\title{
Intrathecal injection of bupivacaine on the contents of excitatory and inhibitory amino acids of spinal cord in rats
}

\author{
Lei Qinghong ${ }^{1}$, Ma Hanxiang ${ }^{1}$, Zhang Yonghai', Yang Fan², Zheng Junwei², Gu Yang ${ }^{2}$ \\ ${ }^{1}$ Department of Anaesthesiology, General Hospital of Ningxia Medical University, Ningxia \\ Yinchuan, China \\ ${ }^{2}$ Department of Anaesthesiology, Ningxia Medical University, Ningxia Yinchuan, China
}

\section{Background and Goal of Study:}

Neuraxal anaesthesia combined general anesthesia has been widely used in anaesthesia practice due to its good properties. Our previous study found that the spinal and epidural anesthesia had sedative effect, but its mechanism still not clear. We hypothesize the sedation of subarachnoid block is related to the influence of bupivacaine on the release of excitatory and inhibitory amino acid in the spinal cord.

\section{Materials and methods:}

24 male Sprague-Dawley rats were randomly divided into three groups: control group (group C, $\mathrm{n}=8$ ), Saline group (group NS, $\mathrm{n}=8$ ), $0.5 \%$ bupivacaine group (group $\mathrm{B}, \mathrm{n}=8$ ). And the rats were intrathecally administrated 25 microliter of saline and 25 microliter of $0.5 \%$ Bupivacaine in the group NS and group B, respectively. Ten minutes after injection, the rats were sacrificed and removed lamina and took out the spinal cord, then placed the spinal cord below thoracic vertebra (T10) in the vials of $1.8 \mathrm{ml}$. Numbered the samples and preserved them in the -80 degrees freezer. The contents of excitatory amino acid aspartate (Asp) and glutamate (Glu) and inhibitory amino acid $\gamma$ aminobutyric acid (GABA) and glycine (Gly) in the rat spinal cord were detected by using the automatic amino acid analyzer (German, S-433D).

\section{Results and discussion:}

The content of GABA in the rats spinal cord in group B $(0.65 \pm 0.10) \quad$ microgram $/ \mathrm{mg}$ were significantly increased comparing with group $\mathrm{C}$ $(0.48 \pm 0.12)$ microgram $/ \mathrm{mg}$ and group NS $(0.49 \pm 0.12)$ microgram $/ \mathrm{mg}(\mathrm{P}<0.01)$;
The content of glycine (Gly) in the rats spinal cord in group B $(1.13 \pm 0.20) \quad$ microgram $/ \mathrm{mg}$ were significantly increased comparing with group $\mathrm{C}$ $(0.91 \pm 0.16) \mathrm{microgram} / \mathrm{mg}$ and group NS $(0.76 \pm 0.13)$ microgram $/ \mathrm{mg}(\mathrm{P}<0.05, \mathrm{P}<0.01)$; however, the content of glutamate (Glu) in the rats spinal cord in group B $(0.91 \pm 0.16) \quad$ microgram $/ \mathrm{mg}$ were significantly decreased comparing with group $\mathrm{C}$ $(1.07 \pm 0.12) \mathrm{microgram} / \mathrm{mg}$ and group NS $(1.14 \pm 0.67)$ microgram $/ \mathrm{mg} \quad(\mathrm{P}<0.05, \mathrm{P}<0.01)$. The sedative effect of subarachnoid block may be related to the changes of excitatory and inhibitory amino acid in the spinal cord.

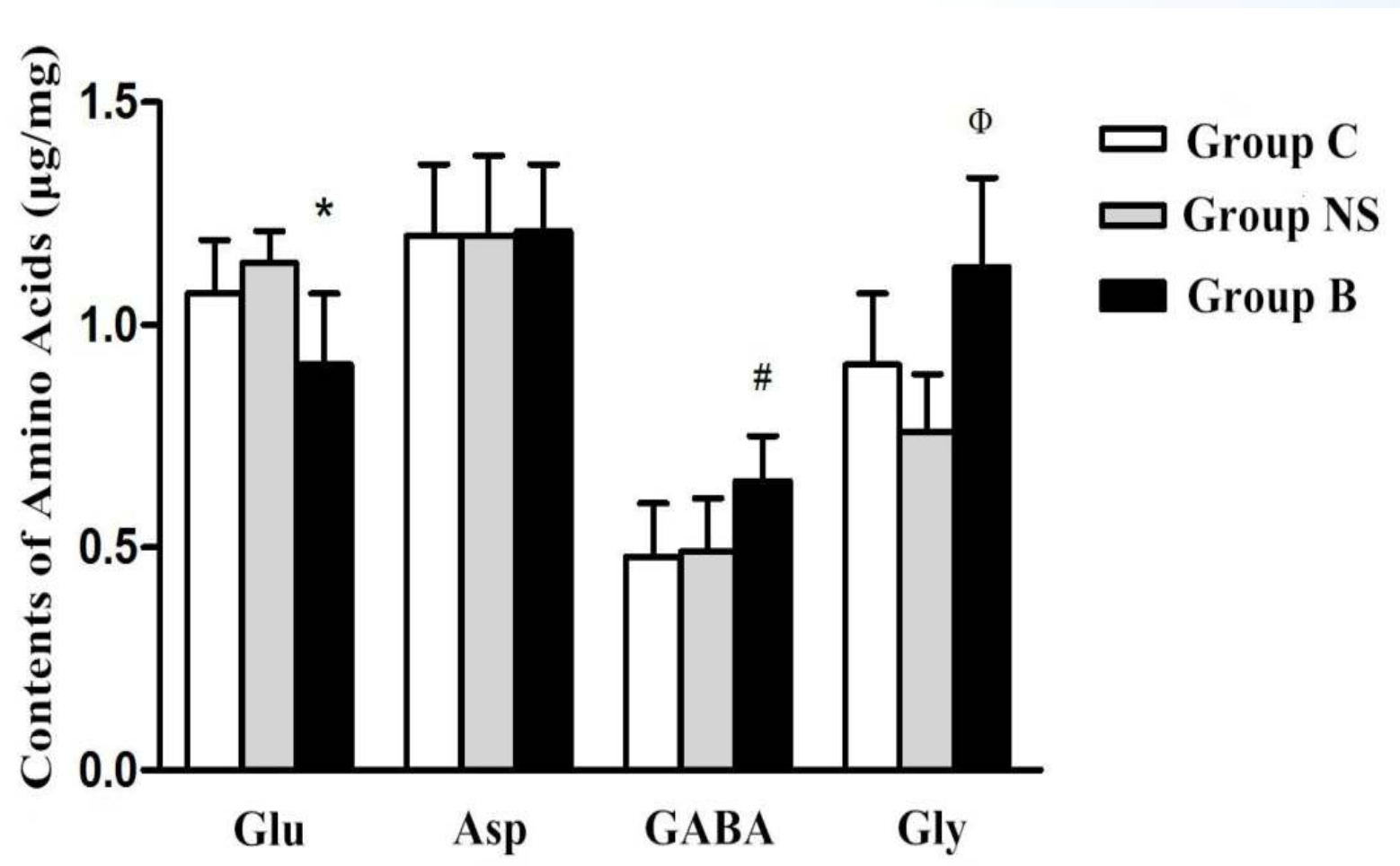

\section{Conclusion:}

Bupivacaine spinal anesthesia can increase the contents of inhibitory amino acid (GABA and Gly) and decrease the contents of excitatory amino acid glutamate. 